\title{
Low Complexity Amplify and Forward Relaying without Channel Amplitude Estimation
}

\author{
Diomidis S. Michalopoulos, Athanasios S. Lioumpas and George K. Karagiannidis \\ Department of Electrical and Computer Engineering \\ Aristotle University of Thessaloniki, Greece \\ Email: \{dmixalo, alioumpa, geokarag\}@auth.gr
}

\begin{abstract}
We propose a low-complexity amplify and forward relaying scheme that avoids channel amplitude estimations by combining the signals associated with the individual relays into an equal gain combiner (EGC). An approximate performance analysis of such scheme is conducted using the well-known moments-based approach. Additionally, motivated by the so-called EGC's combining loss, we propose an algorithmic method that sorts out the weakest relayed branches (in a long-term sense), aiming at activating only the relays that substantially contribute to the overall system performance.
\end{abstract}

\section{INTRODUCTION}

Cooperative diversity has been proposed as an alternative means of achieving diversity, by utilizing spatially distributed terminals that forward the information sent by a source terminal to a destination one. Its operation is based upon the concept of taking full advantage of the wireless networks' distributed nature, by treating the terminals involved as cooperative entities; that is to say, as entities that have both the right to ask help from partner terminals in order to improve their own quality of service (QoS), as well as the duty to contribute to partners' demands when they asked for. As a result, such a cooperative concept is expected to play an important role to future generation wireless communications systems, promising higher QoS and data rates, while keeping the corresponding power consumptions at considerably lower levels.

Following the pioneering work of [1], many papers in the literature deal with the concept of relaying transmissions, either by proposing novel relaying protocols or by conducting a performance analysis of already proposed schemes (see e.g., [2]- [7]). For the case where more than one relays are available, the majority of published works consider either maximal ratio combining (MRC) or some sort of selection combining (SC) of the signals received at the destination. In [3]- [4], for example, the authors arrived at some approximate results for the symbol error probability assuming amplify and forward (AF) relaying and MRC at the destination, whereas in [7] closedform expressions for the outage probability for the case of decode and forward (DF) relaying and MRC at the destination were derived. In [5]- [6] the authors proposed a method that selects the relay with the strongest instantaneous link (either the end-to-end or the relay-destination one) by utilizing appropriate feedback sent by the destination, corresponding thus to a distributed SC model. All the above schemes, however, require that the destination terminal performs a complete channel amplitude estimation of all the incoming links; what is more, is that for the case of AF relaying the destination needs to know the source-relay channels as well, in order to combine the corresponding signals according to their end-to-end (i.e., source-relay-destination) channel conditions.

To this end, in this paper we propose a low-complexity AF cooperative diversity scheme that completely avoids continuous channel amplitude estimation by attributing equal weights to all channels involved and then adding them coherently, i.e., employing coherent distributed equal gain combining (DEGC). An approximate performance analysis of DEGC in terms of bit error probability (BEP) is presented, which demonstrate that, as expected, DEGC performs somewhere between the distributed implementations of MRC and SC. Additionally, motivated by the so-called EGC's combining loss, i.e., the fact that overincreasing the number of branches in an EGC would degrade (instead of enhancing) the overall performance, we propose a method which sorts out the weak (on average) branches during the initialization stage and activates only those that substantially contribute to the overall performance.

The outline of the paper is as follows. Section II describes the DEGC system model, while the the moments of the combiner's output signal to noise ratio (SNR) are presented in Section III. In Section IV, the performance of the proposed system is examined in terms of BEP. In Section $\mathrm{V}$ an algorithmic method for the rejection of the unnecessary relays is proposed. Finally, concluding remarks are presented in Section VI.

\section{SYSTEM MODEL}

We consider the typical cooperative diversity scenario where a source node $S$ communicates with a destination one, $D$, via $L$ relays denoted by $R_{i}, i=1, \ldots, L$. All relays are assumed to operate in the half-duplex AF mode, i.e., they simply amplify the received signal without demodulating it; also, in order to satisfy causality, they do not receive and transmit simultaneously, but on different time slots. For this reason, each transmission period is divided into two sub-slots: In the former, the source broadcasts its message to the relays; at the same time, the destination listen the same signal and it buffers it for later use. In the latter sub-slot, only the relays forward to the destination the previously received signal, each one using a separate orthogonal channel.

All channels involved (i.e., $S-D, S-R_{i}, R_{i}-D$ ) are assumed to experience independent, flat and slow Rayleigh fading, not 
necessarily identically distributed, resulting in exponentially distributed SNRs. We denote with $\gamma_{0}$ the instantaneous SNR of the direct, $S$ - $D$ channel. The instantaneous end-to-end SNR of the virtual $S-R_{i}-D$ channel $^{1}$ is given by [8, eq. (16)]

$$
\gamma_{i}=\frac{\gamma_{S R_{i}} \gamma_{R_{i} D}}{C_{i}+\gamma_{R_{i} D}}
$$

where $\gamma_{S R_{i}}, \gamma_{R_{i} D}$ represent the instantaneous SNR of the $S-R_{i}$ and $R_{i}-D$ link, respectively; $C_{i}$ is a constant, which depends only on the channel's statistics and is related to the amplification gain $G_{i}$ through

$$
C_{i}=\frac{P_{R_{i}}}{G_{i}^{2} N_{0}}
$$

where $P_{R_{i}}$ stands for the relay's transmission power and $N_{0}$ represents the additive white Gaussian noise power, which is assumed constant in all links. Throughout this paper we assume that the relays have access to the average receiver-end channel state information (CSI), being thus able to employ the semiblind fixed gain proposed in [8] in order to limit the relay's average transmission power; hence, $C_{i}$ is expressed as [8]

$$
C_{i}=\frac{\bar{\gamma}_{S R_{i}} \exp \left(-1 / \bar{\gamma}_{S R_{i}}\right)}{E_{1}\left(1 / \bar{\gamma}_{S R_{i}}\right)},
$$

where $E_{n}(\cdot)$ is the exponential integral function of order $n$ defined in [9, eq. (5.1.1)] and the overbar ( $)$ denotes expectation. We emphasize that, as shown in [8], such gain assumption leads to a very similar performance to that of instantaneous CSI-assisted gain relays, notwithstanding the latter's higher complexity.

As soon as the destination receives the signal replicas incident from all the terminals involved, it combines them into an equal gain combiner (EGC), yielding an output SNR that has the form of [10, eq. (9.94)]

$$
\gamma_{\text {out }}=\frac{\left(\sum_{i=0}^{L} \sqrt{\gamma_{i}}\right)^{2}}{L+1} \text {. }
$$

Recall that EGC does not require instantaneous channel amplitude estimation; this, in conjunction with the time-invariable gains that the relays are assumed to employ (i.e., fixed relay gains independent of the instantaneous front-end channel), eliminates the need for continuous channel monitoring at either the relays or the destination, hence it corresponds to a lowcomplexity relaying scheme.

\section{Moments OF THE COMBINER's OUTPUT SNR}

Lemma 1: For every real $z$ and $i \in\{1, \ldots, L\}$, it holds

$$
E\left\langle\gamma_{i}^{z}\right\rangle=\left(\bar{\gamma}_{S R_{i}}\right)^{z} \Gamma^{2}(z+1) U\left(z, 0, \frac{C_{i}}{\bar{\gamma}_{R_{i} D}}\right),
$$

where $E\langle\cdot\rangle$ denotes expectation, $U(\cdot, \cdot, \cdot)$ is the Tricomi confluent hypergeometric function $U$ defined in [9, eq. (13.1.3)] and $\Gamma(\cdot)$ is the Gamma function [11, eq. (8.310.1)].

\footnotetext{
${ }^{1}$ Throughout this paper, the virtual $S-R_{i}-D$ channels are also referred to as branches, since they actually form a relaying multibranch system.
}

Proof: Averaging $\gamma_{i}^{z}$ over the distributions of $\gamma_{S R_{i}}$ and $\gamma_{R_{i} D}$ we obtain

$$
\begin{aligned}
& E\left\langle\gamma_{i}^{z}\right\rangle=\int_{0}^{\infty} \int_{0}^{\infty}\left(\frac{x y}{C_{i}+y}\right)^{z} \frac{e^{-\frac{x}{\bar{\gamma} S R_{i}}}}{\bar{\gamma}_{S R_{i}}} \frac{e^{-\frac{y}{\bar{\gamma}_{R_{i} D}}}}{\bar{\gamma}_{R_{i} D}} d x d y \\
= & {\left[\int_{0}^{\infty} x^{z} \frac{e^{-\frac{x}{\bar{\gamma}_{S R_{i}}}}}{\bar{\gamma}_{S R_{i}}} d x\right]\left[\int_{0}^{\infty}\left(\frac{y}{C_{i}+y}\right)^{z} \frac{e^{-\frac{y}{\bar{\gamma}_{R_{i} D}}}}{\bar{\gamma}_{R_{i} D}} d y\right] }
\end{aligned}
$$

Using [11, eq. (3.381.4)], the first integral in (6) yields

$$
\int_{0}^{\infty} x^{z} \frac{e^{-\frac{x}{\bar{\gamma}_{S R_{i}}}}}{\bar{\gamma}_{S R_{i}}} d x=\bar{\gamma}_{S R_{i}}^{z} \Gamma(z+1)
$$

Using successively the variable-change $\omega=y / C_{i}$ and integration by parts, the second integral in (6) yields

$$
\begin{gathered}
\int_{0}^{\infty}\left(\frac{y}{C_{i}+y}\right)^{z} \frac{e^{-\frac{y}{\bar{\gamma}_{R_{i} D}}}}{\bar{\gamma}_{R_{i} D}} d y=\int_{0}^{\infty}\left(\frac{\omega}{\omega+1}\right)^{z} \frac{C_{i} e^{-\frac{C_{i} \omega}{\bar{\gamma}_{R_{i} D}}}}{\bar{\gamma}_{R_{i} D}} d \omega \\
=\left[\left(\frac{\omega}{\omega+1}\right)^{z} e^{-\frac{C_{i} \omega}{\bar{\gamma} R_{i} D}}\right]_{0}^{\infty}-\int_{0}^{\infty} \frac{z \omega^{z-1}}{(\omega+1)^{z+1}} e^{-\frac{C_{i} \omega}{\bar{\gamma}_{R_{i} D}}} d \omega \\
=\frac{\Gamma(z+1)}{\Gamma(z)} \int_{0}^{\infty} \frac{\omega^{z-1}}{(\omega+1)^{z+1}} e^{-\frac{C_{i} \omega}{\bar{\gamma}_{R_{i} D}}} d \omega \\
=\Gamma(z+1) U\left(z, 0, \frac{C_{i}}{\bar{\gamma}_{R_{i} D}}\right)
\end{gathered}
$$

where we have used [9, eq. (6.1.15)] and [9, eq. (13.2.5)] to obtain the two last lines of (8). The proof is completed by substituting (7) and (8) into (6).

As a corollary to Lemma 1 , we may notice that the $n$th moment of $\gamma_{i}$ is obtained directly from (5) by setting $z=n$, where $n \in \mathbb{N}$; the use of such corollary, however, is beyond the scope of this paper. We also note that the moments of $\gamma_{0}$ are the moments of a Rayleigh distributed channel, already given in [10, eq. (2.9)].

Theorem 1: The $n$th moment of the SNR at the destination's combiner output is given by

$$
\begin{aligned}
\mu_{n}= & \frac{(2 n) !}{(L+1)^{n}} \sum_{\substack{n_{0}, n_{1}, \ldots, n_{L}=0 \\
n_{0}, n_{1}+\ldots+n_{L}=2 n}}^{2 n} \frac{\Gamma\left(\frac{n_{0}}{2}+1\right) \bar{\gamma}_{0}^{n_{0} / 2}}{n_{0} !} \\
& \prod_{i=1}^{L} \frac{\bar{\gamma}_{S R_{i}}^{n_{i} / 2} \Gamma^{2}\left(\frac{n_{i}}{2}+1\right) U\left(\frac{n_{i}}{2}, 0, \frac{C_{i}}{\bar{\gamma}_{R_{i} D}}\right)}{n_{i} !}
\end{aligned}
$$

Proof: Taking expectations in (4), we obtain

$$
\mu_{n}=E\left\langle\gamma_{\text {out }}^{n}\right\rangle=E\left\langle\left[\frac{1}{L+1}\left(\sum_{i=0}^{L} \sqrt{\gamma_{i}}\right)^{2}\right]^{n}\right\rangle .
$$


Applying the multinomial theorem, (10) yields

$$
\begin{gathered}
\mu_{n}=\frac{(2 n) !}{(L+1)^{n}} \sum_{\substack{n_{0}, n_{1}, \ldots, n_{L}=0 \\
n_{0}, n_{1}+\ldots+n_{L}=2 n}}^{2 n} \frac{\prod_{i=0}^{L} E\left\langle\gamma_{i}^{\frac{n_{i}}{2}}\right\rangle}{\prod_{i=0}^{L} n_{i} !} \\
=\frac{(2 n) !}{(L+1)^{n}} \sum_{\substack{n_{0}, n_{1}, \ldots, n_{L}=0 \\
n_{0}, n_{1}+\ldots+n_{L}=2 n}}^{2 n} \frac{\Gamma\left(\frac{n_{0}}{2}+1\right) \bar{\gamma}_{0}^{\frac{n_{0}}{2}} \prod_{i=1}^{L} E\left\langle\gamma_{i}^{\frac{n_{i}}{2}}\right\rangle}{\prod_{i=0}^{L} n_{i} !}
\end{gathered}
$$

where we have used the fact that the channels fade independently with one another and that $E\left\langle\gamma_{0}^{k}\right\rangle=\Gamma(k+1) \bar{\gamma}_{0}^{k}, k \in$ $\mathbb{R}$, obtained by averaging $\gamma_{0}^{k}$ over the exponential probability density function (PDF) of $\gamma_{0}$ and then using the integral representation of the gamma function [9, eq. (6.1.15)]. Consequently, (9) is straightforwardly derived by applying Lemma 1 in (11).

\section{Performance Analysis}

1) Average Output SNR: The average output SNR is derived from (9) by setting $n=1$. Using the fact that $U(0,0, z)=1$ and $U(1,0, z)=e^{z} E_{2}(z), z \in \mathbb{R}[9$, Table 13.6], we may obtain the average DEGC output SNR as

$$
\begin{aligned}
& \bar{\gamma}_{\text {out }}=\frac{1}{L+1}\left[\sum_{i=1}^{L} \bar{\gamma}_{S R_{i}} e^{\frac{C_{i}}{\bar{\gamma}_{i} D}} E_{2}\left(\frac{C_{i}}{\bar{\gamma}_{R_{i} D}}\right)+2 \sum_{i=1}^{L} \sum_{\substack{j=1 \\
j \neq i}}^{L}\right. \\
& \sqrt{\bar{\gamma}_{S R_{i}} \bar{\gamma}_{S R_{j}}} \Gamma^{4}\left(\frac{3}{2}\right) U\left(\frac{1}{2}, 0, \frac{C_{i}}{\bar{\gamma}_{R_{i} D}}\right) U\left(\frac{1}{2}, 0, \frac{C_{j}}{\bar{\gamma}_{R_{j} D}}\right) \\
& \left.+\bar{\gamma}_{0}+2 \sqrt{\bar{\gamma}_{0}} \sum_{i=1}^{L} \sqrt{\bar{\gamma}_{S R_{i}}} \Gamma^{3}\left(\frac{3}{2}\right) U\left(\frac{1}{2}, 0, \frac{C_{i}}{\bar{\gamma}_{R_{i} D}}\right)\right] .
\end{aligned}
$$

2) Bit Error Probability $(B E P)$ : Let $f_{\sigma}(\cdot)$ represent the PDF of the sum $\sum_{i=0}^{L} \sqrt{\gamma_{i}}$. The average BEP can be evaluated by averaging the conditional BEP over $f_{\sigma}(\cdot)$, yielding

$$
P_{b}=\int_{0}^{\infty} \frac{\operatorname{erfc}(x / \sqrt{L+1})}{2} f_{\sigma}(x) d x,
$$

where $\operatorname{erfc}(\cdot)$ is the complementary error function [11, eq. (8.2.50)]. Unfortunately, $f_{\sigma}(\cdot)$ is not easily tractable since it actually represents the convolution of the PDF of $\sqrt{\gamma_{i}}$; an analytical evaluation of which is cumbersome. For this reason, in the sequel we confine ourselves to an approximate analysis of the BEP, by using the well-known moments-based approach.

\section{A. An Approximate MGF-Based Performance Analysis}

1) Padé Approximants to the Output SNR: The moment generating function (MGF) of the $\gamma_{\text {out }}$ is defined as

$$
\mathcal{M}_{\gamma_{\text {out }}}(s)=E\left\langle e^{s \gamma_{\text {out }}}\right\rangle \text {. }
$$

Alternatively, using the well-known Taylor series, we may express the MGF of $\gamma_{\text {out }}$ in a infinite-term series form, i.e.,

$$
\mathcal{M}_{\gamma_{\text {out }}}(s)=\sum_{n=0}^{\infty} \frac{E\left\langle\gamma_{\text {out }}^{n}\right\rangle s^{n}}{n !}=\sum_{n=0}^{\infty} \frac{\mu_{n} s^{n}}{n !} .
$$

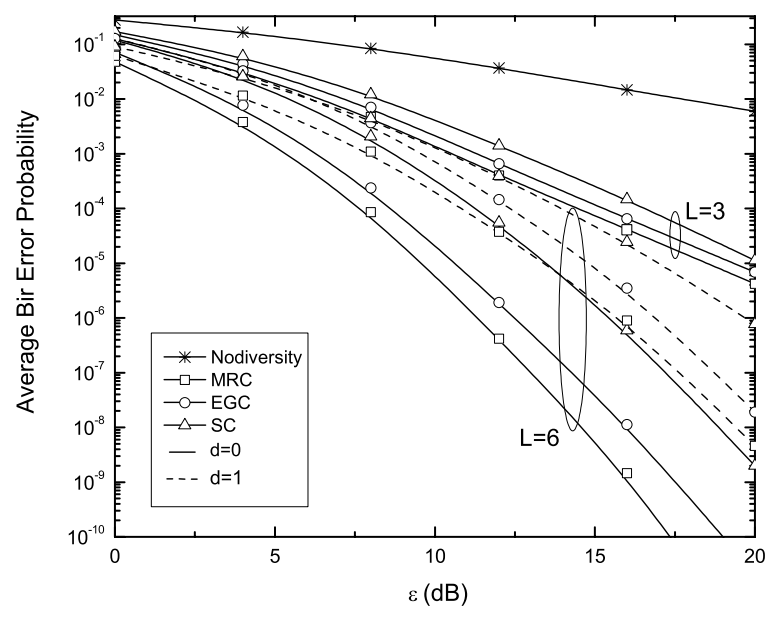

Fig. 1. BEP of the DEGC, the distributed MRC and distributed SC scheme versus $\varepsilon$, for several values of $d$, assuming BPSK modulation

Due to the fact that the convergence of the series in (14) cannot be always assured, we confine ourselves to obtaining an approximation of $\mathcal{M}_{\gamma_{\text {out }}}(s)$ by utilizing the Padé approximation method. Such method is widely used in several scientific fields, serving as an alternative means of approximating series similar to that in (14) where practically only few coefficients are known and the series converges too slowly or diverges [12], [13].

A Padé approximant is a rational function approximation to $\mathcal{M}_{\gamma_{\text {out }}}(s)$, namely $\mathcal{P}_{[A / B]}(s)$, whose nominator and denominator are of order $A$ and $B$, respectively, and whose power expansion is identical with the $A+B$ order expansion of $\mathcal{M}_{\gamma_{\text {out }}}(s)$, i.e.,

$$
\mathcal{P}_{[A / B]}(s) \equiv \frac{\sum_{j=1}^{A} \alpha_{i} s^{i}}{1+\sum_{j=1}^{B} \beta_{i} s^{i}}=\sum_{n=0}^{A+B} \frac{\mu_{n} s^{n}}{n !}+O\left(s^{A+B+1}\right),
$$

where $O\left(s^{A+B+1}\right)$ is the remainder after the truncation of the infintie-term series to that of order $A+B$. Consequently, only the first $A+B$ moments of the combiner's output SNR are needed in order to construct $\mathcal{P}_{[A / B]}(s)$ and therefore to obtain an approximation for $\mathcal{M}_{\gamma_{\text {out }}}(s)$. In the sequel, we use only the subdiagonal Padé approximants $\mathcal{P}_{[A / A+1]}(s)$, since it is only for that approximants that the convergence rate and the uniqueness can be assured [12]. We note that Padé approximants are available in the majority of the well-known mathematical software packages, such as MATHEMATICA and MAPLE.

2) BEP Performance: Having found an approximate expression for $\mathcal{M}_{\gamma_{\text {out }}}(s)$, the BEP of the DEGC system can be derived by using the MGF-based approach presented in [10, ch. 1]. In Fig. 1, we present the BEP of the DEGC scheme for BPSK modulation for some values of $L$, when operating over independent but not necessarily identically distributed Rayleigh fading channels, which follows from the fact that in practical applications the relays are randomly distributed in space, resulting in generally non-identical fading statistics. Hence, in order to have an insight into the performance of the proposed 


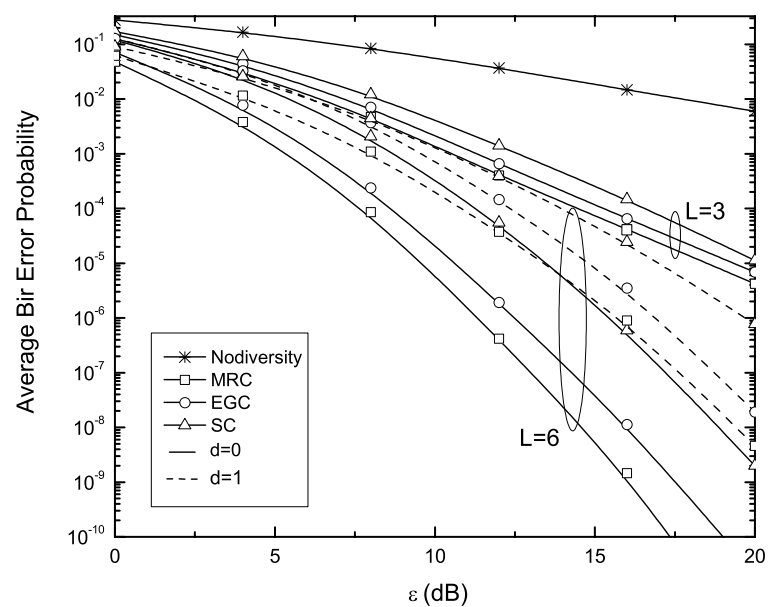

Fig. 2. Average output SNR, of the DEGC, the distributed MRC and distributed SC scheme versus the number of participating relays, $L$, for several values of the decay factor $d$

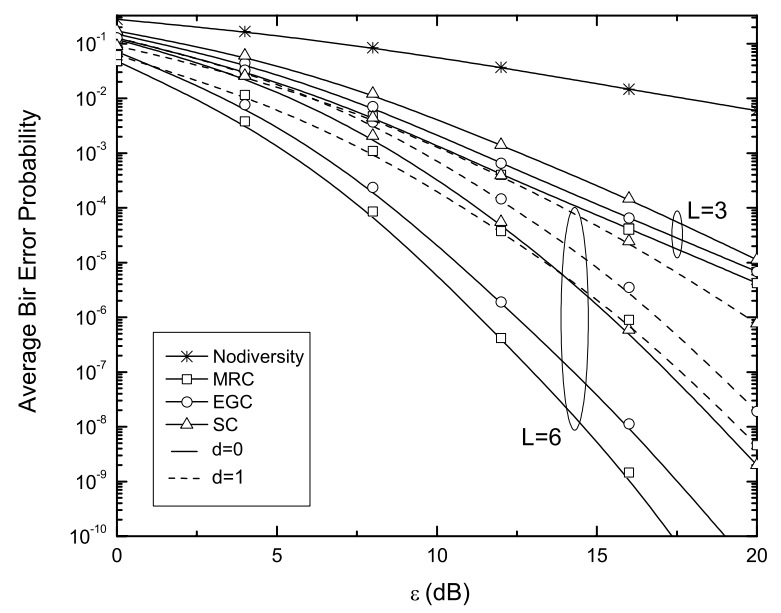

Fig. 3. BEP of the DEGC, the distributed MRC and distributed SC scheme versus the number of participating relays, $L$, for several values of the decay factor $d$, assuming BPSK modulation

scheme over a wide range of disparatness on the average channel conditions, the average SNRs of the $S-R_{i}$ and $R_{i}-D$ links are considered to follow an exponential profile with decay factor denoted by $d$ and mean values denoted by $\varepsilon$ (equal with one another), i.e., $\bar{\gamma}_{S R_{i}}=\alpha e^{-d(i-1)}, \quad \bar{\gamma}_{R_{i} D}=\alpha e^{-d(i-1)}$, $i \in\{1, \ldots, L\}$, where $\alpha=L \varepsilon\left(e^{d}-1\right) /\left[\left(e^{d L}-1\right) e^{-d(L-1)}\right]$ is an appropriately chosen constant so that $\varepsilon$ does not depend on $L$. For comparison purposes, the corresponding error probabilities for the distributed MRC and SC models are also depicted, assuming the same exponential SNR profile. As expected, the DEGC performance is better than that of SC and worse than that of MRC. Moreover, for low values of $d$ DEGC performs closely to MRC, whereas for higher values of $d$ (i.e., higher disparateness among the corresponding SNRs) the relative DEGC performance worsens and becomes asymptotically comparable to that of SC. We also note that the BEP curves corresponding to the DEGC scheme were obtained by using the $\mathcal{P}_{[8 / 9]}(s)$ approximant, since it was found that the results for this Padé order closely match the simulations ones, i.e., they match at the fifth significant digit.

\section{A Statistics-Based Algorithmic Method for SORTING OUT THE WEAKEST BRANCHES}

Figs. 2 and 3 respectively depict the average output SNR $\bar{\gamma}_{\text {out }}$ and the BEP of the DEGC scheme versus the number of available relays, $L$, for BPSK modulation. As it is shown in these figures, the performance of the DEGC scheme as a function of $L$ appears to reach a floor point, i.e., after a certain value of $L$, adding more relays to the system does not lead to a performance enhancement, but to higher complexity and yet to specrtal inefficiency, since each one of the participating relay is attributed with its own orthogonal channel. Generally speaking, the main drawback of employing an EGC at the destination is the incurred combining loss, which becomes more evident as the disparateness among the available branches' SNRs increases [10, ch. 9.3]. This stems from the fact that all the available branches are equi-weighted; hence in cases where some of them are in deep fade, combining the weak branches together with the strong ones results in performance degradation, since the weak branches would actually contribute more to enhancing the noise than the signal during the combining process.

Consequently, it is apparent that the terminals that finally participate in the relaying process need to be appropriately selected, so that each one's activation would also entail a performance enhancement, apart from the inevitable complexity and bandwidth consumption increase. Towards this end, in this section we propose an algorithmic method that selects the relays according to their average channel conditions and then sorts out the weakest ones.

\section{A. The Algorithmic Relay Selection Method}

Due to the similarities of the DEGC receiver with the classic EGC one, our relay selection method is similar to that presented in [14], where a long term statistical criterion was used for the rejection of the weakest diversity branches in EGC receivers. It should be noted that although a long term criterion could theoretically involve any order of the SNR statistics, we utilize only the first and second moments of the combined SNR, which is a more practical realization of such a criterion. Thus, a possible algorithmic implementation for the DEGC could be the following.

1) Estimate the mean $E\left\langle\gamma_{i}\right\rangle$ and the variance $E\left\langle\left(\gamma_{i}-E\left\langle\gamma_{i}\right\rangle\right)^{2}\right\rangle$ of the end-to-end SNR for each relayed branch, using a long training sequence at the initialization stage.

2) Calculate the long term performance metric for each branch, say $\zeta_{i}$, for $i=1, \ldots L$, and sort them in increasing (decreasing) order (e.g., $\zeta_{[1]} \leq \zeta_{[2]} \leq \ldots \leq$ $\zeta_{[L]}$, where $\zeta_{[i]}$ is the ordered $\left.\zeta_{i}\right)$. At this step the set of selected branches, termed as $\mathcal{S}_{l}^{*}$, includes only the branch 
This full text paper was peer reviewed at the direction of IEEE Communications Society subject matter experts for publication in the ICC 2008 proceedings.

with the minimum (maximum) $\zeta$ (i.e., $\zeta_{[1]}$ ) and the output performance metric, $\zeta_{\text {out }}$, equals to the minimum $\zeta$ (i.e., $\left.\zeta_{\text {out }}=\zeta_{[1]}\right)$.

3) Add the branch with the next smaller (greater) $\zeta$ to the set $\mathcal{S}_{l}^{*}$ and calculate the output performance metric, $\zeta_{\text {out }}$. If the resulted $\zeta_{\text {out }}$ is greater (smaller) than the previous one, exclude the last added branch and go to step 5. The output metric can be evaluated using the closed form formulas presented in (9).

4) Repeat step 3 until the maximum number of branches has been reached.

5) Equally weight and sum the signals from the branches in $\mathcal{S}_{l}^{*}$.

6) Repeat the steps 1-5 if the channels' long-term statistics change.

\section{B. Generalized Channel Quality Estimation Index (GCQEI)}

Extensive simulation experiments showed that an appropriate metric for the case of DEGC could be a modified version of the Channel Quality Estimation Index (CQEI) proposed in [14]. The latter metric was proposed for quantifying the quality of point-to-point communications, where no relaying takes place. In cooperative relaying, however, the end-to-end SNR variance around its mean value is higher, because of the relaying channels' cascaded nature, hence for the DEGC case the metric's dependence on the SNR variance needs to be modified. Toward this direction, we propose the generalized CQEI (GCQEI) defined as

$$
G C Q E I=\frac{A F_{i}^{\nu}}{\bar{\gamma}_{i}}
$$

where $A F_{i}$ stands for the amount of fading of the $i$ th branch defined as [10, eq. (1.27)]

$$
A F_{i}=\frac{E\left\langle\gamma_{i}^{2}\right\rangle-\left(E\left\langle\gamma_{i}\right\rangle\right)^{2}}{\left(E\left\langle\gamma_{i}\right\rangle\right)^{2}},
$$

and $\nu$ is a positive real constant. We note that similarly to [14], GCQEI represents a minimization criterion. Through the parameter $\nu, \mathrm{GCQEI}$ offers the possibility to control the relative sensitivity of this metric to the SNR variance around its mean value, compared to the mean value itself. As a result, the parameter $\nu$ can actually determine the percentage of relays that are activated out of the set of available ones, which in practical applications can be adjusted according to the desired trade-off between the performance and the number of activated relays. In Table I we present the proposed algorithm's behavior for several values of $\nu$ (with respect to the number of available branches, $L$ ). As it is shown in this Table, for relatively small values of the decay factor $d$ DEGC attains similar or better BEP compared to the all-participate EGC, although the activated relays are fewer. We also notice that, as expected, the effectiveness of the proposed algorithm in rejecting the weakest relayed channels is more evident for larger valued of $d$.
TABLE I

PERCENTAGE OF PARTICIPATING RELAYS AND BEP OF THE PROPOSED AND THE ALL-PARTICIPATE DEGC SCHEME FOR SEVERAL VALUES OF $\nu$ AND BPSK MODULATION

\begin{tabular}{|c|c|c|c|}
\hline$v / L$ & \multicolumn{3}{|c|}{$d=0.75$} \\
\hline & Active relays & $B E R_{G C Q E I}$ & $B E R_{E G C}$ \\
\hline 0.1 & $5 / 7$ & $2.4138810^{-7}$ & $1.2365410^{-7}$ \\
\hline 0.3 & $6 / 7$ & $1.0642310^{-7}$ & $1.2365410^{-7}$ \\
\hline 0.5 & $7 / 7$ & $1.2365410^{-7}$ & $1.2365410^{-7}$ \\
\hline 0.7 & $7 / 7$ & $1.2365410^{-7}$ & $1.2365410^{-7}$ \\
\hline 0.9 & $7 / 7$ & $1.2365410^{-7}$ & $1.2365410^{-7}$ \\
\hline 1.1 & $7 / 7$ & $1.2365410^{-7}$ & $1.2365410^{-7}$ \\
\hline \multicolumn{4}{|c|}{$d=1.25$} \\
\hline 0.1 & $3 / 7$ & $2.1917510^{-5}$ & $1.419610^{-5}$ \\
\hline 0.3 & $4 / 7$ & $7.6992710^{-6}$ & $1.419610^{-5}$ \\
\hline 0.5 & $5 / 7$ & $7.0748910^{-6}$ & $1.419610^{-5}$ \\
\hline 0.7 & $5 / 7$ & $7.0748910^{-6}$ & $1.419610^{-5}$ \\
\hline 0.9 & $5 / 7$ & $7.0748910^{-6}$ & $1.419610^{-5}$ \\
\hline 1.1 & $5 / 7$ & $7.0748910^{-6}$ & $1.419610^{-5}$ \\
\hline
\end{tabular}

\section{ACKNOWLEDGMENT}

This work was conducted within the framework of the Reinforcement Program of Human Research Manpower (PENED'03), partially funded by the E.U.-European Social Fund $(75 \%)$ and the Greek Ministry of Development (25\%).

\section{REFERENCES}

[1] J. N. Laneman, D. N. C. Tse, and G. W. Wornell, "Cooperative diversity in wireless networks: Efficient protocols and outage behavior," IEEE Trans. Inform. Theory, vol. 50, pp. 3062-3080, Dec. 2004.

[2] M. O. Hasna and M. S. Alouini, "End-to-end performance of transmission systems with relays over Rayleigh fading channels," IEEE Trans. Wireless Commun., vol. 2, pp. 1126-1131, Nov. 2003.

[3] A. Ribeiro, X. Cai, and G. Giannakis, "Symbol error probabilities for general cooperative links," IEEE Trans. Wireless Commun., vol. 4, pp. 1264-1273, May 2005.

[4] P. A. Anghel and M. Kaveh, "Exact symbol error probability of a cooperative network in a Rayleigh-fading environment," IEEE Trans. Wireless Commun., vol. 3, pp. 1416-1421, Sept. 2004.

[5] A. Bletsas, A. Khisti, D. P. Reed, and A. Lippman, "A simple cooperative diversity method based on network path selection," IEEE J. Selec. Areas Commun., vol. 24, pp. 659-672, Mar. 2006.

[6] E. Beres and R. Adve, "On selection cooperation in distributed networks," in Proc. of Conference on Information Sciences and Systems (CISS 06), Princeton, NJ, Mar. 2006.

[7] N. C. Beaulieu and J. Hu, "A closed-form expression for the outage probability of decode-and-forward relaying in dissimilar rayleigh fading channels," IEEE Commun. Let., vol. 10, pp. 813-815, Dec. 2006.

[8] M. O. Hasna and M. S. Alouini, "A performance study of dual-hop transmissions with fixed gain relays," IEEE Trans. Wireless Commun., vol. 3, pp. 1963-1968, Nov. 2004.

[9] M. Abramovitz and I. A. Stegun, Handbook of Mathematical Functions with Formulas, Graphs, and Mathematical Tables, 9th ed. New York: Dover, 1972.

[10] M. K. Simon and M.-S. Alouini, Digital Communication over Fading Channels, 2nd ed. New York: Wiley, 2005.

[11] I. S. Gradshteyn and I. M. Ryzhik, Table of Integrals, Series, and Products, 6th ed. New York: Academic, 2000.

[12] G. A. Baker and P. Graves-Morris, Padé Approximants. Cambridge Universiy Press, 1996.

[13] H. Amindavar and J. A. Ritcey, "Padé approximations of probability density functions," vol. 30, pp. 416-424, Apr. 1994.

[14] A. S. Lioumpas, G. K. Karagiannidis, and A. C. Iossifides, "Channel quality estimation index (CQEI): A long-term performance metric for fading channels and an application in EGC receivers," IEEE Trans. Wireless. Commun., vol. 6, pp. 3315-3322, Sept. 2007. 